\title{
Composición y propiedades cerámicas de las pizarras de la Zona Centro Ibérica del Macizo Ibérico Meridional (Norte de Jaén, España)
}

\author{
M. VÁZQUEZ ${ }^{1}$, J. JIMÉNEZ-MILLÁN ${ }^{1}$, C. SÁNCHEZ-JIMÉNEZ ${ }^{2}$, J. PARRAS ${ }^{3}$ \\ ${ }^{1}$ Departamento de Geología, Fac. Ciencias Experimentales, Univ. Jaén, 23071 Jaén \\ ${ }^{2}$ Lab. de Mineralogía Aplicada, Fac. de Químicas, Univ. Castilla-La Mancha, 13071 Ciudad Real \\ ${ }^{3}$ Escuela Universitaria de Magisterio, 13071 Ciudad Real
}

\begin{abstract}
En este trabajo se ha evaluado la aplicabilidad para fabricar materiales cerámicos de las pizarras del Macizo Ibérico de la provincia de Jaén. Los principales componentes de estas pizarras son illita, caolinita, cuarzo y feldespatos, con menores contenidos de clorita, pirofilita, paragonita e interestratificado clorita/vermiculita. Estos materiales tienen altos contenidos de alúmina (17-21\%), mientras el oxido de hierro varía en un amplio intervalo (1.33-9.21\%). Los análisis granulométricos muestran que más del $90 \%$ de las partículas son $<20 \mu \mathrm{m}$. La mayoría de estos materiales son sólo potencialmente apropiados para el moldeo por presión, debido a la baja plasticidad relacionada con el alto contenido en cuarzo y feldespatos. Los valores de contracción lineal y absorción de agua de estas pizarras, cocidas alrededor de $1000^{\circ} \mathrm{C}$, sugieren que pueden ser apropiadas para fabricar algunos tipos de baldosas porosas, tales como mayólica o cottoforte. Además, este estudio ha revelado que estos materiales cocidos entre $1150^{\circ} \mathrm{C}$ y $1250^{\circ} \mathrm{C}$ pueden ser usados para elaborar gres rojo y gres blanco.
\end{abstract}

Palabras clave: Macizo Ibérico, Pizarras paleozoicas, vitrificación, contracción lineal, absorción de agua.

\section{Composition and ceramic properties of shales from the Central-Iberican Zone of Southern Iberian Massif (Northern Jaen, Spain)}

The suitability of shale materials from the Iberian Massif in the Jaén province (Spain) to make ceramic materials has been evaluated. The main components of these shales are illite, kaolinite, quartz and feldspars, with lesser amounts of chlorite, pyrophyllite, paragonite and chlorite/vermiculite mixed-layers. The materials contain high amounts of alumina (17-21\%), and iron oxide vary over a wide range (1.33-9.21\%). Grain-size analysis shows that more than $90 \%$ is $<20 \mu \mathrm{m}$. Most of these materials are only potentially suitable for shaping by pressing, due to the low plasticity related with the high quartz and feldspar content. The linear shrinkage and water absorption values of the materials fired at around $1000^{\circ} \mathrm{C}$ suggest they might be suitable for making some types of porous tiles like majolica or cottoforte. In addition, this study has revealed that the Iberian shales from norther Jaén can be used to make red and white stoneware tiles by firing at temperatures between $1150{ }^{\circ} \mathrm{C}$ and $1250{ }^{\circ} \mathrm{C}$.

Keywords: Iberian Massif, Palaeozoic shales, vitrifification, linear shrinkage, water absorption.

\section{INTRODUCCIÓN}

Las arcillas illítico-cloríticas e illítico-caoliníticas son las materias primas comúnmente usadas para la fabricación de gres $(1,2)$. Las mezclas de estas arcillas con materiales complementarios, de naturaleza cuarzo-feldespática, son empleadas para mejorar su comportamiento durante el ciclo de producción cerámico, especialmente en el caso de los productos fabricados mediante monococción. La producción de pavimentos y revestimientos de color rojo requiere la utilización de materias primas cuyo contenido en óxido de hierro se encuentre entre el 6 y $8 \%$ (1). Son numerosas las materias primas que presentan estas características composicionales, entre las cuales podemos destacar las pizarras del distrito cerámico de Sassuolo, las cuales son usadas ampliamente para la fabricación de gres rojo (3). Sin embargo, desde el comienzo de los años ochenta, se ha dado una preferencia significativa en la elaboración de piezas de color claro obtenidas a partir de materias primas con bajos contenidos en óxido de hierro (4), las cuales derivan, en su mayoría, de rocas alteradas. En el norte de la provincia de Jaén existen extensos afloramientos de pizarras y rocas cuarzo-feldespáticas que pertenecen a la parte meridional de la Zona Centro-Ibérica del Macizo Ibérico. Algunas de estas pizarras han sufrido procesos de alteración relacionados con la actividad tectónica, el magmatismo, la actividad hidrotermal y la formación de los yacimientos de metales base en el distrito minero de Linares-La Carolina. En este trabajo se ha realizado una caracterización composicional y tecnológica de estas pizarras paleozoicas para evaluar su aplicabilidad en la fabricación de pavimentos y revestimientos cerámicos.

\section{CONTEXTO GEOLÓGICO}

El área estudiada se encuentra en la unidad para-autóctona de la parte meridional de la Zona Centro-Ibérica del Macizo Ibérico (5). El basamento de la zona estudiada está constituido por una sucesión de pizarras y rocas metavolcánicas, de edades comprendidas entre el Ordovícico y el Carbonífero. Las muestras han sido tomadas en dos áreas: la Sierra de Andujar, formada por rocas del Ordovícico Inferior al Carbonífero Inferior, y el área de la Carolina, constituida por rocas del Ordovícico al Carbonífero Inferior. Para este trabajo se tomaron dieciséis muestras de pizarras paleozoicas de seis formaciones geológicas: pizarras Silúricas (PS) y pizarras de los Estratos Orthis (PE) del área de La Carolina, y pizarras Pochico (PP), pizarras Botella (PB), pizarras Cantera (PC), y pizarras Chavera $(\mathrm{PCH})$, del área de la Sierra de Andújar. 


\section{TÉCNICAS EXPERIMENTALES}

Todas las muestras han sido sometidas a análisis mineralógicos y químicos para determinar sus características composicionales. La composición mineralógica ha sido determinada mediante el uso de difracción de rayos X (XRD, Siemens D5000 con filtro de Ni, radiación Cu Ka: $35 \mathrm{KV}$ y $34 \mathrm{~mA}$ ) y microscopía electrónica de barrido (SEM, Jeol JSM-5800) con microanálizador (EDS, Oxford Link). Los análisis mineralógicos cualitativos se han llevado a cabo en la muestra total molida y en la fracción $<2 \mu \mathrm{m}$. La mineralogía de arcillas está determinada en agregados orientados (natural, calentados a $550^{\circ} \mathrm{C}$ durante $2 \mathrm{~h}$ y solvatados en etilenglicol y dimetilsulfóxido). Los análisis semi-cuantitativos se han realizado siguiendo el método estándar usando los parámetros calculados para el difractómetro Siemens D-5000 de la Universidad de Jaén y datos de otros autores (6-9). La microtextutura de las muestras y los análisis de los filosilicatos han sido estudiados mediante el uso de SEM. Los análisis químicos de los elementos mayoritarios han sido realizados usando espectrometría de fluorescencia de rayos X (Philips PW 1404/10) mediante perlas de fusión.

Los ensayos de laboratorio han consistido en una molienda en húmedo (mortero de porcelana durante 10 minutos), secado, análisis granulométricos mediante dispersión de rayo láser (Galai CIS-1), desagregación y humectación (4-6\% agua), prensado a $300 \mathrm{Kg} / \mathrm{cm}^{2}$, secado durante 24 h a $110^{\circ} \mathrm{C}$ y cocción en un horno eléctrico a $200^{\circ} \mathrm{C}$ y $\mathrm{T}_{\text {máx }}$ de $2 \mathrm{~h}$. La contracción lineal y los limites de Atterberg (limite liquido, LL, y limite plástico, PL, según UNE 7-377-75 y UNE 103-104-93, respectivamente) están medidos en las piezas secas. Por otra parte, la contracción lineal, la absorción de agua (EN 99), la porosidad, la densidad aparente y el color (D.R. Lange, Microcolor-LMC con luz normalizada D 65) han sido medidos en las piezas cocidas. También ha sido usada la XRD para determinar las fases de alta temperatura en las piezas cocidas y SEM y microscopia electrónica de transmisión (TEM, Philips CM-20) han sido usadas para estudiar las fases minerales formadas y su microestruturas.

\section{CARACTERÍSTICAS COMPOSICIONALES}

\subsection{Composición mineralógica}

Los datos de XRD mostrados en la Tabla I revelan que las muestras estudiadas son ricas en filosilicatos (53-77\%), cuarzo (6-16\%) y feldespatos (7-14\%). El mayor contenido en filosilicatos pertenece a las muestras de PS (77\%). Las muestras de PCH y PP se caracterizan por la presencia de pequeñas cantidades de hematites. La illita es el principal componente de la asociación de filosilicatos (36-86\%). Las muestras de PS y PE tienen los contenidos más altos de caolinita (41 y 29\%, respectivamente), mientras las muestras de PC y PCH están caracterizadas por la presencia de esmectita. También se encontraron cantidades poco importantes de clorita (3-15\%) y, de forma subordina$\mathrm{da}$, pirofilita, esmectita y paragonita. El estudio de SEM revela la textura foliada predominante en las rocas estudiadas (Fig. 1 A). También han sido observados porfidoblastos de andalucita en las muestras de PS. En estas muestras, la caolinita aparece remplazando a los cristales de andalucita y dispersa en la matriz filosilicatada (Fig. 1 B).
TABLA I. COMPOSICIÓN MINERALÓGICA DE LAS PIZARRAS PALEOZOICAS DEL NORTE DE LA PROVINCIA DE JAÉN (\% EN PESO).

\begin{tabular}{|l|c|c|c|c|c|c|c|c|c|c|}
\hline & \multicolumn{4}{|c|}{ Muestra total } & \multicolumn{5}{|c|}{ Fracción $<2 \mu \mathrm{m}$} \\
\hline & Phy & Fds & Qtz & Hem & Prl & Kln & Ill & Chl & Pg & Sm \\
\hline PS & 77 & 8 & 15 & - & - & 41 & 46 & $<5$ & 10 & - \\
\hline PE & 67 & 11 & 22 & - & - & 29 & 66 & $<5$ & - & - \\
\hline PP & 57 & 7 & 31 & 6 & 36 & 6 & 54 & $<5$ & - & - \\
\hline PB & 59 & 15 & 26 & - & - & - & 86 & $<5$ & 10 & - \\
\hline PC & 53 & 11 & 36 & - & - & $<5$ & 64 & 7 & - & 25 \\
\hline PCH & 70 & 12 & 16 & $<5$ & - & 10 & 36 & 15 & $<5$ & 34 \\
\hline
\end{tabular}

Phy: filosilicatos. Fds: feldespatos. Qtz: cuarzo. Hem: hematites. Prl: pirofilita. Kln: caolinita. Ill: illita. Chl: clorita. Pg: paragonita. Sm: esmectita.
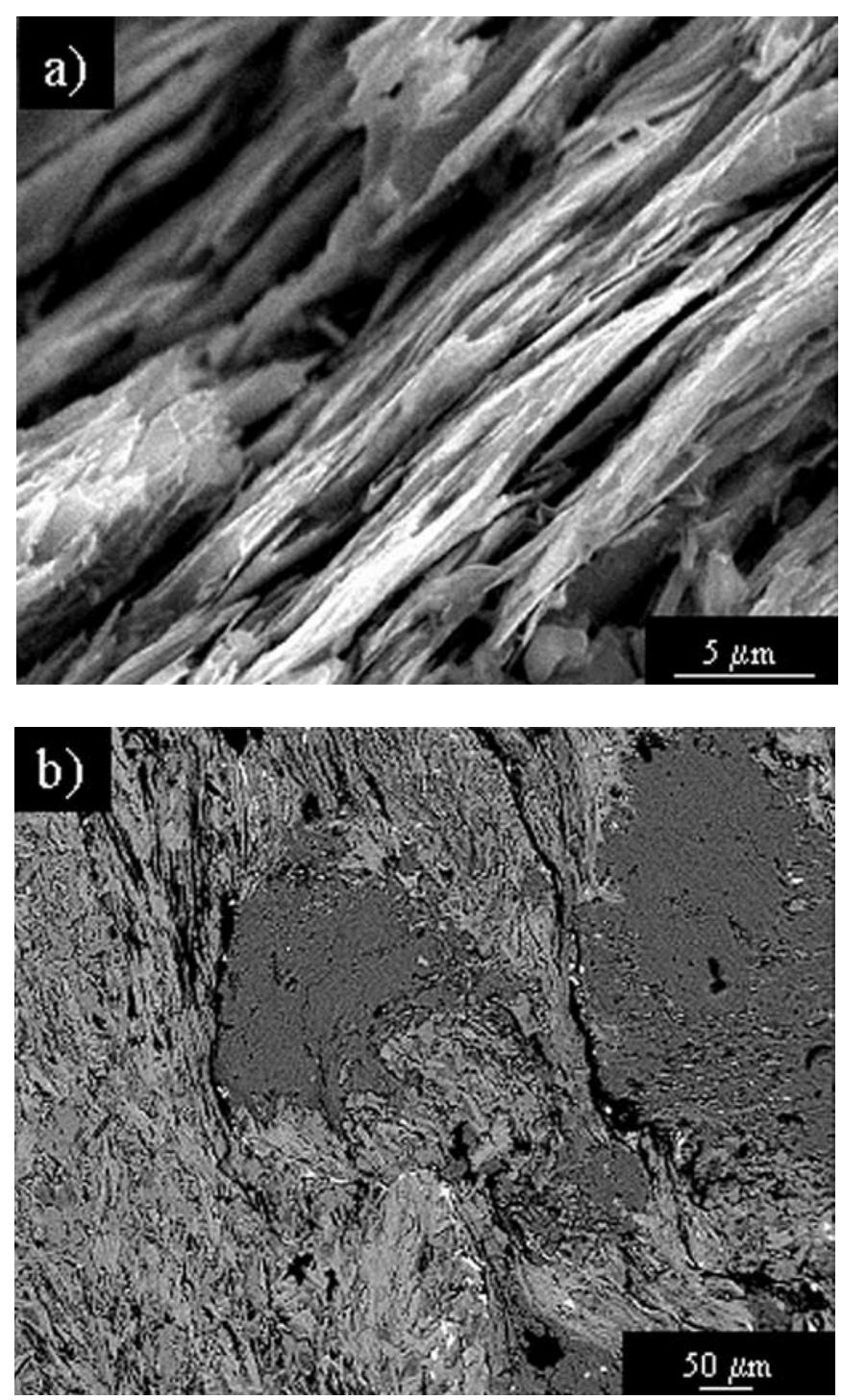

Figura 1. A) Imagen de SEM mostrando la textura foliada de las pizarras estudiadas. B) Imagen de BSE en la que se observan porfidoblastos de andalucita remplazados por cristales de andalucita en una matriz illítico-caolinítica. 


\subsection{Composición química}

La composición química de las pizarras estudiadas (Tabla II), de acuerdo con su composición mineralógica, presenta altas cantidades de sílice (57-66\%), alúmina (17-21\%) y $\mathrm{K}_{2} \mathrm{O}$ (1.77-4.53\%), estando éste último asociado a la presencia de micas y feldespatos. El contenido en $\mathrm{Fe}_{2} \mathrm{O}_{3}$ varía entre $1.33 \%$ en la muestra PS (donde no existe hematites y el contenido en illita es bajo) y el 5.62 y el $9.21 \%$, debidos a la presencia de hematites (PP y PCH) o illita fengítica (PE, PB, PC).

\subsection{Propiedades tecnológicas}

\section{3. 1. GRANULOMETRÍA}

La tabla III y la Fig. 2 muestran la variabilidad de la distribución granulométrica de las pizarras paleozoicas molidas. La fracción dominante es la situada entre 2 y $20 \mu \mathrm{m}$, la cual varía entre el 55 y el 65\%. El contenido en la fracción arcilla varía entre el $26 \%$ y el $45 \%$, mientras que la fracción gruesa es muy poco abundante, alcanzando valores máximos del $9 \%$ en PE y PP.

TABLA II. COMPOSICIÓN QUÍMICA DE LAS PIZARRAS PALEOZOICAS DEL NORTE DE LA PROVINCIA DE JAÉN (\% EN PESO).

\begin{tabular}{|l|l|l|l|l|l|l|l|l|}
\hline & $900^{\circ} \mathrm{C}$ & $950^{\circ} \mathrm{C}$ & $1000^{\circ} \mathrm{C}$ & $1050^{\circ} \mathrm{C}$ & $1100^{\circ} \mathrm{C}$ & $1150^{\circ} \mathrm{C}$ & $1200^{\circ} \mathrm{C}$ & $1250^{\circ} \mathrm{C}$ \\
\hline PS & $(79,10,14)$ & $(80,10,13)$ & $(80,11,12)$ & $(78,11,12)$ & $(76,9,13)$ & $(74,7,13)$ & $(71,4,12)$ & $(69,2,9)$ \\
\hline PE & $(55,15,17)$ & $(55,15,16)$ & $(55,14,15)$ & $(52,13,12)$ & $(44,11,9)$ & $(40,6,6)$ & $(42,5,7)$ & $(42,4,8)$ \\
\hline PP & $(59,18,15)$ & $(60,18,15)$ & $(60,17,14)$ & $(58,16,13)$ & $(53,17,14)$ & $(48,16,11)$ & $(42,11,4)$ & $(42,3,4)$ \\
\hline PB & $(61,19,24)$ & $(61,22,25)$ & $(58,25,25)$ & $(52,25,23)$ & $(44,19,17)$ & $(42,10,6)$ & $(39,5,4)$ & $(45,4,6)$ \\
\hline PC & $(59,17,22)$ & $(59,20,23)$ & $(57,23,22)$ & $(49,24,21)$ & $(42,17,14)$ & $(40,8,6)$ & $(40,2,2)$ & $(47,2,4)$ \\
\hline PCH & $(61,16,21)$ & $(60,20,23)$ & $(56,24,22)$ & $(52,23,20)$ & $(44,16,14)$ & $(42,8,5)$ & $(40,2,4)$ & $(45,5,7)$ \\
\hline
\end{tabular}

TABLA III. DisTRIBUCIÓN GRANULOMÉTRICA DE LAS MATERIAS PRIMAS ESTUDIADAS (\% EN PESO).

\begin{tabular}{|l|l|l|l|l|l|l|l|l|l|l|l|}
\hline & $\mathrm{SiO}_{2}$ & $\mathrm{Al}_{2} \mathrm{O}_{3}$ & $\mathrm{Fe}_{2} \mathrm{O}_{3}$ & $\mathrm{MnO}$ & $\mathrm{MgO}$ & $\mathrm{CaO}$ & $\mathrm{Na}_{2} \mathrm{O}$ & $\mathrm{K}_{2} \mathrm{O}$ & $\mathrm{TiO}_{2}$ & $\mathrm{P}_{2} \mathrm{O}_{5}$ & L.o.I \\
\hline PS & 57.06 & 28.37 & 1.33 & 0.01 & 0.46 & 0.71 & 0.62 & 2.99 & 1.31 & 0.04 & 4.47 \\
\hline PE & 63.85 & 16.65 & 6.73 & 1.37 & 0.44 & 0.09 & 0.24 & 4.53 & 0.93 & 0.24 & 4.37 \\
\hline PP & 66.09 & 19.91 & 9.21 & - & 0.19 & 0.04 & 0.35 & 1.77 & 1.11 & 0.10 & 4.39 \\
\hline PB & 58.36 & 20.73 & 7.05 & 0.06 & 1.89 & 0.29 & 1.18 & 3.98 & 1.02 & 0.14 & 4.52 \\
\hline PC & 67.26 & 15.94 & 5.62 & 0.04 & 1.65 & 0.42 & 1.85 & 2.65 & 0.85 & 0.19 & 3.21 \\
\hline PCH & 59.30 & 20.60 & 6.68 & 0.07 & 1.95 & 0.34 & 1.16 & 4.49 & 1.03 & 0.19 & 4.11 \\
\hline
\end{tabular}

L.o.I: Perdida por calcinación $\left(1000^{\circ} \mathrm{C}\right)$.

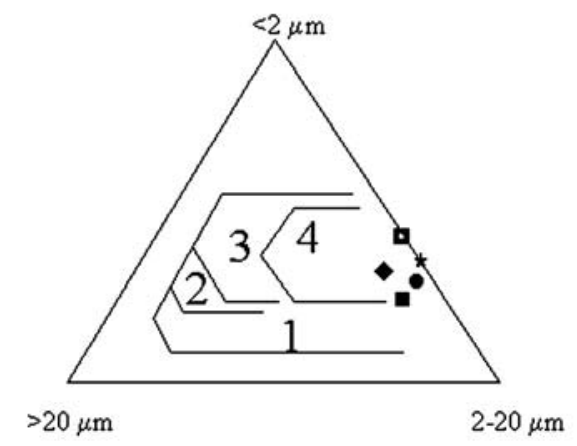

- $\mathrm{PS} \backsim \mathrm{PE} \bullet \mathrm{PP} \square \mathrm{PB} \star \mathrm{PC} \circ \mathrm{PCH}$

Figura 2. Distribución granulométrica de las materias primas paleozoicas estudiadas del norte de la provincia de Jaén en el diagrama de Winkler (10). (1) ladrillos comunes (2) ladrillos perforados verticalmente (3) tejas y bloques ligeros (4) bloques y ladrillos perforados.

\section{3. 2. PLASTICIDAD Y PROPIEDADES DE MOLDEO}

Los análisis mostrados en las Figs. 3 y 4 indican que las pizarras del norte de la provincia de Jaén tienen generalmente un comportamiento poco plástico, debido a los bajos valores del limite liquido (menor del $40 \%$ excepto para PS, donde éste es del $45 \%)$ e índice plástico $(<37 \%)$.

\section{3. 3. PROPIEDADES DE COCCIÓN}

La variación de las propiedades cerámicas durante los ensayos de cocción realizados entre 900 y $1250{ }^{\circ} \mathrm{C}$ no es uniforme. Los datos de contracción lineal y de absorción de agua en función de la temperatura de cocción han sido representados en la Fig. 5 (página siguiente). Para la mayoría de las muestras estudiadas, las propiedades cerámicas de las arcillas son bastante estables en el intervalo de temperatura entre $950{ }^{\circ} \mathrm{C}$ y $1000^{\circ} \mathrm{C}$, donde la absorción de agua varía entre el 16 y el $25 \%$ y la contracción lineal oscila entre -1 y $0.4 \%$. A partir de los $1000{ }^{\circ} \mathrm{C}$, se produce una reducción drástica de la absorción de agua y un incremento súbito de la contracción lineal. Este cambio de tendencia puede relacionarse con el comienzo de la vitrificación.

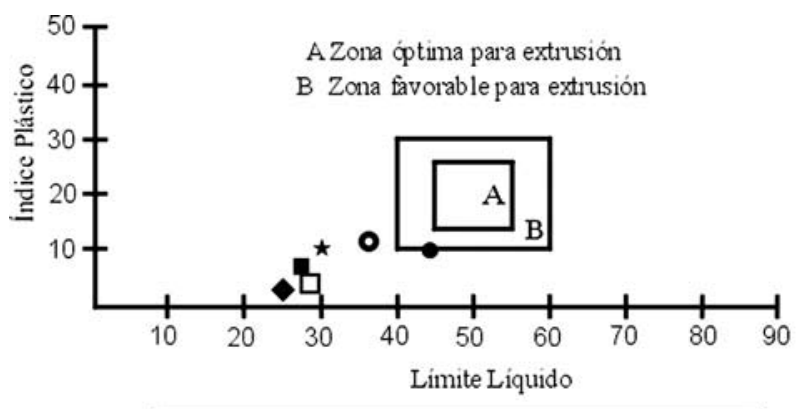

- $\mathrm{PS} \quad \mathrm{PE} \diamond \mathrm{PP} \quad \square \mathrm{PB} \star \mathrm{PC}$ OPCH

Figura 3. Diagrama de Casagrande de las materias primas paleozoicas estudiadas del norte de la provincia de Jaén.

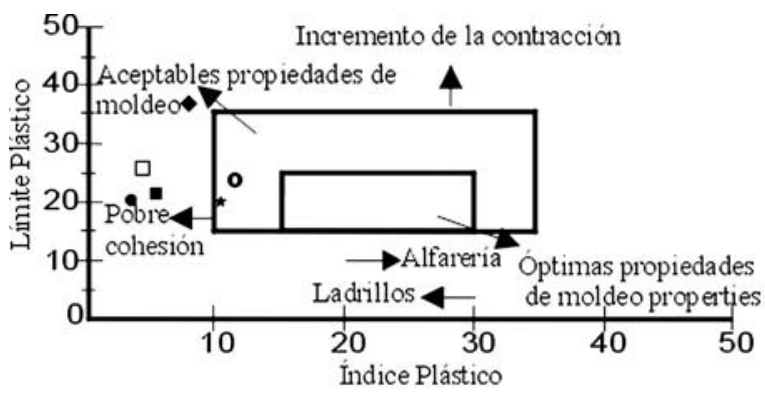

- $\mathrm{PS} \backsim \mathrm{PE} \diamond \mathrm{PP} \square \mathrm{PB} \star \mathrm{PC}$ OPCH

Figura 4. Diagrama de manejabilidad de las materias primas paleozoicas estudiadas del norte de la provincia de Jaén. 
A)

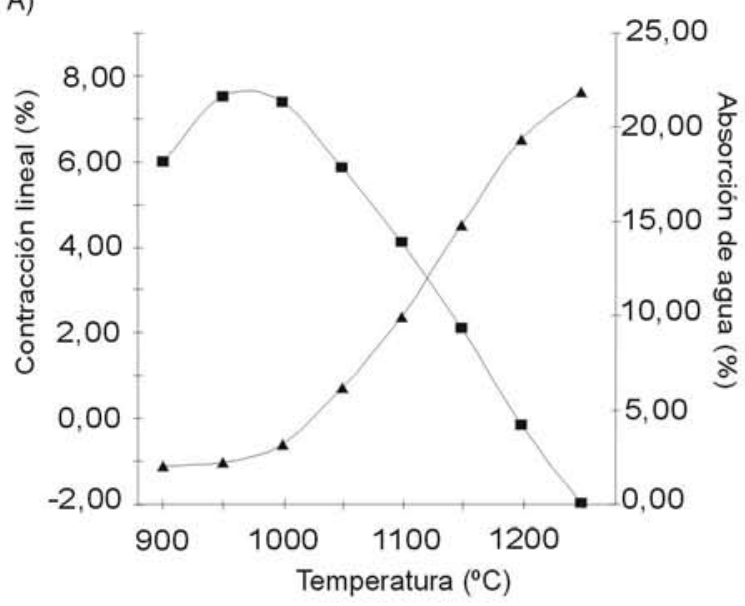

C)

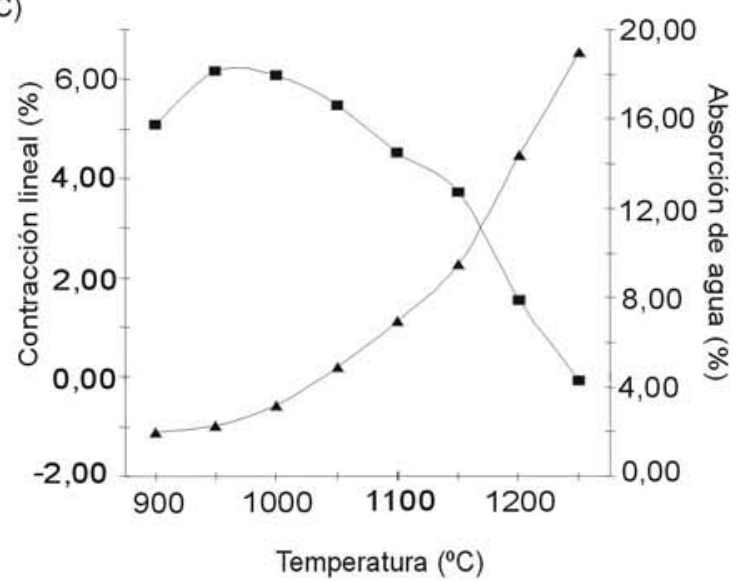

E)

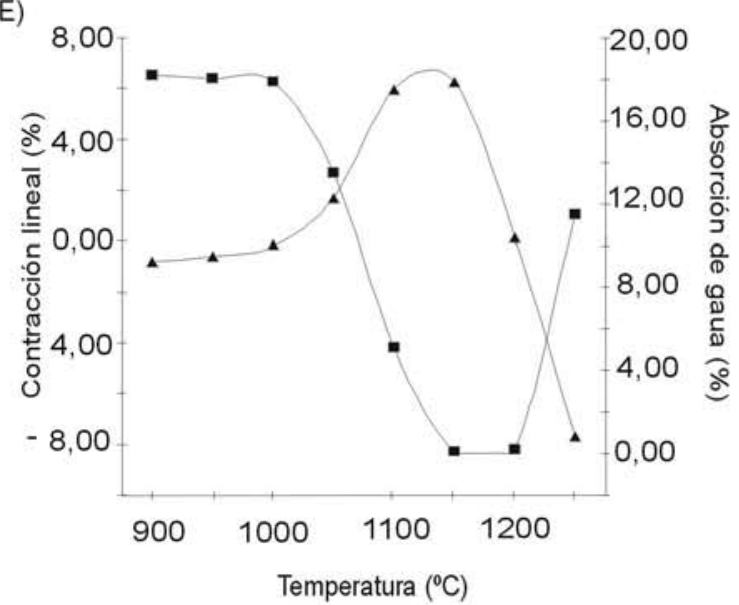

B)
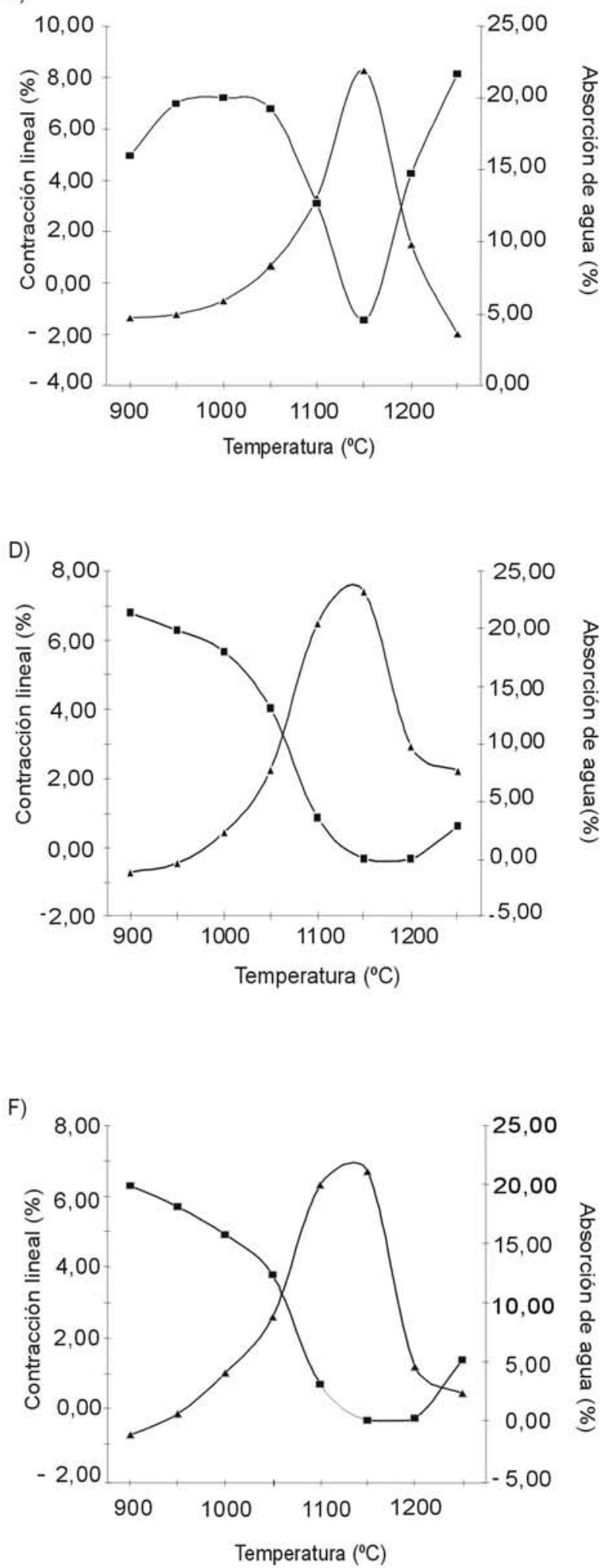

\section{- $\boldsymbol{\Delta}$ - Contracción lineal 는 Absorción de agua}

Figure 5. Ensayos de cocción de las materias primas paleozoicas estudiadas del norte de la provincia de Jaén: a) PS, b) PE, c) PP, d) PB, e) PC, f) PCH 
Las muestras PB, PC, PCH tienen un comportamiento bastante similar, alcanzando valores de absorción de agua de alrededor del $5 \%$ a $1100{ }^{\circ} \mathrm{C}$ y casi nulos entre $1150{ }^{\circ} \mathrm{C}$ y 1200 ${ }^{\circ} \mathrm{C}$ Debe subrayarse que los valores más altos de contracción lineal de estos materiales (alrededor del 7\%) se alcanzan a una temperatura de $1150{ }^{\circ} \mathrm{C}$. A partir de esta temperatura, se observa una reducción drástica de esta propiedad, oscilando entre el $0 \%$ y el 3\% a $1200{ }^{\circ} \mathrm{C}$ (Figs. 5 D, E, F). También se ha encontrado un comportamiento similar en los materiales de PE, donde la vitrificación se desarrolla en un intervalo de temperatura más estrecho situado alrededor de los $1150{ }^{\circ} \mathrm{C}$.

Las muestras PS y PP son las pizarras más refractarias. Las piezas cerámicas obtenidas de las muestras de PP no se encuentran completamente vitrificadas a $1250^{\circ} \mathrm{C}$ (la mayor temperatura usada en este trabajo), presentando un valor de absorción de agua del 4\% (Fig. 5F). Finalmente, las muestras de PS se caracterizan por alcanzar la máxima contracción lineal (alrededor del 8\%) cuando la absorción de agua es cero a $1250{ }^{\circ} \mathrm{C}$ (Fig. $5 \mathrm{~A}$ ).

Con respecto al color de cocción (Tabla IV), debe subrayarse que el color claro desarrollado a altas temperaturas en la muestra de PS con coordenadas $L^{*}, a, b$ de $(69,2,9)$. Por el contrario, el resto de materiales ensayados presentan colores rojizos.

Los datos de XRD, SEM y TEM de las muestras cocidas revelan la existencia de mullita, hematites, cristobalita, espinela y una fase vítrea. La mullita ha sido detectada desde los 1000 ${ }^{\circ} \mathrm{C}$, su proporción, tamaño, y cristalinidad incrementa con el aumento de la temperatura de cocción. El estudio de TEM revela que a $1250{ }^{\circ} \mathrm{C}$, los cristales de mullita forman un entra-

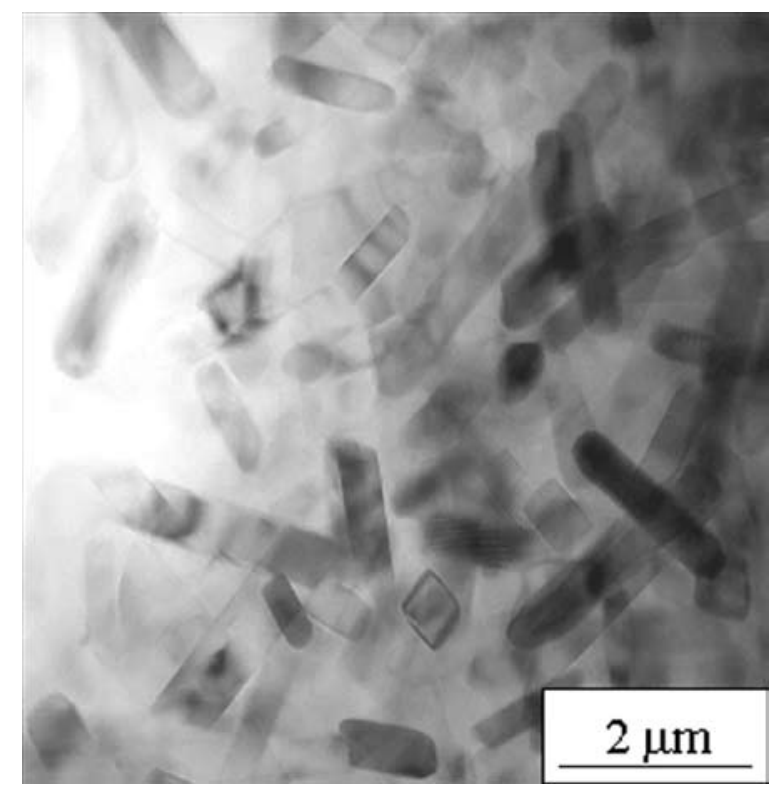

Figura 6. Imagen de TEM de la muestra PS a $1250^{\circ} \mathrm{C}$ mostrando cristales de mullita formando un entramado de agujas.

TABla IV. CoORdenadas CIELAB (L*, A, B) DE LAS MUESTRAS COCIDAS.

\begin{tabular}{|l|c|c|c|}
\hline & $>20 \mu \mathrm{m}$ & $2-20 \mu \mathrm{m}$ & $<2 \mu \mathrm{m}$ \\
\hline PS & 3 & 65 & 32 \\
\hline PE & 9 & 64 & 27 \\
\hline PP & 9 & 56 & 35 \\
\hline PB & - & 55 & 45 \\
\hline PC & - & 63 & 37 \\
\hline PCH & - & 55 & 45 \\
\hline
\end{tabular}

mado de agujas (Fig. 6). Por otra parte, los contenidos en hematites de las piezas cocidas sugieren que parte de la hematites observada se forma desde $\operatorname{los} 1000^{\circ} \mathrm{C}$ a partir de goetita y geles de hierro. La cristalización de cristobalita ha sido observada a $1200{ }^{\circ} \mathrm{C}$ en los materiales de PP. Cantidades traza de espinela han sido observadas en los materiales de PB y PC en el intervalo de temperatura entre $1050{ }^{\circ} \mathrm{C}$ y $1200{ }^{\circ} \mathrm{C}$, lo cual es coherente con el mayor contenido de $\mathrm{MgO}$ que presentan estas muestras. La fase vítrea comienza a desarrollarse desde los $1000{ }^{\circ} \mathrm{C}$, convirtiéndose en el principal componente a partir de los $1150^{\circ} \mathrm{C}$. La fig. 7 muestra la evolución de la microsestructura de las piezas cocidas con el aumento de temperatura en la muestra PB. El estado inicial de vitrificación se observa a $1000^{\circ} \mathrm{C}$ (Fig. 7 A). A esta temperatura, aunque todavía pueden reconocerse los agregados laminares de los minerales de la arcilla, como es el caso de los minerales micáceos, es posible comenzar a identificar algunos filamentos de vidrio dispersos. A $1050^{\circ} \mathrm{C}$ (Fig. 7 B), el porcentaje y el tamaño de los filamentos vitrificados aumenta, dando lugar a una textura caracterizada por la presencia de poros alargados, tales como los descritos previamente por Parras et al. (10) motivada por la coalescencia de los minerales de la arcilla. La vitrificación se completa a $1150{ }^{\circ} \mathrm{C}$, lo cual se manifiesta por la formación de una matriz vítrea continua (Fig. $7 \mathrm{C}$ ). En consonancia con esta textura, a esta temperatura se alcanza el valor mínimo de los valores de absorción de agua. A $1200{ }^{\circ} \mathrm{C}$ (Fig. 7 D) hay un incremento de la porosidad. Una evolución final similar fue observada en las piezas cerámicas de baja porosidad estudiadas por Orts et al. (11).

\section{DISCUSIÓN}

\subsection{Características composicionales}

De acuerdo con Dondi et al. (12), la proporción entre el contenido en filosilicatos, cuarzo y feldespatos es apropiada para la fabricación de productos de cerámica estructural. Además, la asociación de filosilicatos (illita+caolinita+clorita) es compatible con la requerida para fabricar pavimentos y revestimientos cerámicos. Concretamente, los altos contenidos de caolinita de PS sugieren su uso potencial para la fabricación de piezas cerámicas de color claro.

Respecto a la composición química de las pizarras estudiadas, los contenidos en sílice y alúmina son apropiados para la fabricación de productos de cerámica estructural (ver Dondi et al. (12)). Por otra parte, el bajo contenido en hierro de PS revela que estas materias primas podrían ser utilizadas para la fabricación de gres blanco. Finalmente, el contenido en óxido de hierro del resto de las pizarras estudiadas es compatible con el requerido para la elaboración de gres rojo.

\subsection{Propiedades tecnológicas}

Como puede observarse en la figura 2, la granulometría no se ajusta a los campos definidos para las materias primas más frecuentemente utilizadas para la elaboración de productos de cerámica estructural $(13,14)$. Sin embargo, debe subrayarse que debido al predominio de los tamaños finos respecto al grueso, las muestras molidas de pizarras paleozoicas se proyectan en las proximidades del campo definido para piezas perforadas. 

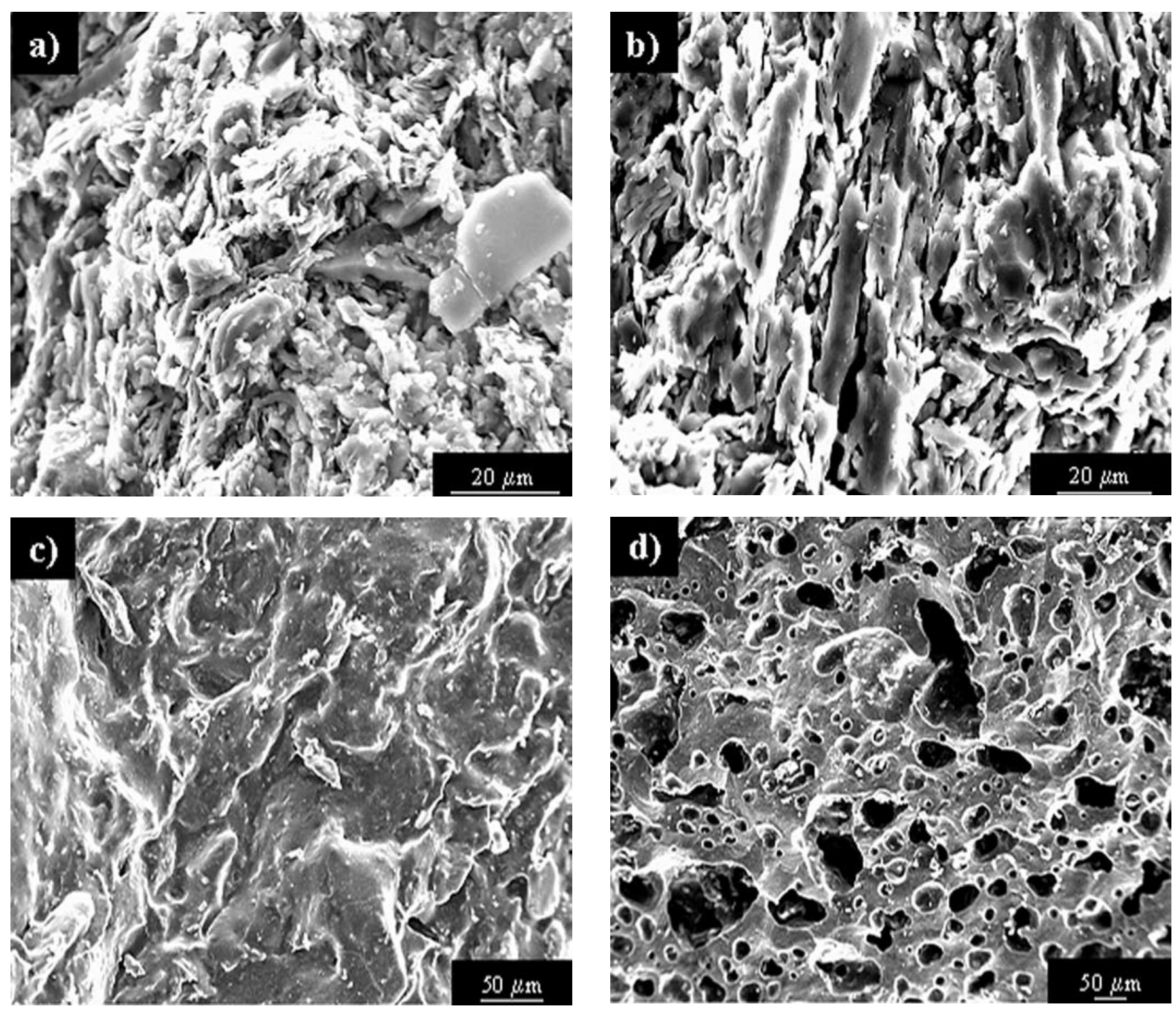

Figura 7. Imágenes de SEM de las piezas cocidas. A) PB a $1000^{\circ} \mathrm{C}$. B) $\mathrm{PB}$ a $1050^{\circ} \mathrm{C}$. C) $\mathrm{PB}$ a $1150{ }^{\circ} \mathrm{C}$. D) $\mathrm{PB}$ a $1200^{\circ} \mathrm{C}$

La plasticidad de las pizarras paleozoicas estudiadas es baja, lo cual está relacionado con el alto contenido en la fracción situada entre 2 y $20 \mu \mathrm{m}$ y la presencia importante de minerales desgrasantes tales como cuarzo y feldespatos. De acuerdo con el diagrama de Casagrande, la mayoría de las pizarras estudiadas son aptas para el moldeo por presión. Sólo la muestra PS tienen la suficiente plasticidad para el moldeo por extrusión (Fig. 3), probablemente como resultado de su mayor contenido en filosilicatos. Sin embargo, de acuerdo con el diagrama de manejabilidad de Bain y Highley (15), las muestras de PC y PCH tienen aceptables propiedades para el moldeado (Fig. 4), lo cual puede ser explicado por su mayor contenido en esmectita.

El comportamiento de las pizarras estudiadas durante la cocción está fuertemente condicionado por las características composicionales de las muestras estudiadas. De esta forma, las muestras (PE, PCI, PB, PC, PCH) con una mineralogía rica en minerales fundentes, tales como esmectita, illita, feldespatos, clorita y paragonita, alcanzan la vitrificación a temperaturas menores. Sin embargo, las muestras PP y PS presentan un comportamiento refractario. Este comportamiento puede ser explicado teniendo en cuenta los bajos contenidos en alcalinos y altos contenidos en sílice y alúmina para la muestra de las PP y a la alta proporción en caolinita para la muestra PS. La influencia de la distribución granulométrica en las propiedades de cocción ha sido observada en las muestras PE y PP. De esta forma, el predominio de la fracción gruesa sobre la fina en las muestras de las PP puede explicar, en parte, los altos valores de absorción de agua a $1250{ }^{\circ} \mathrm{C}(4 \%)$ cuando los valores de contracción lineal máximos son moderados $(6 \%)$. En el caso de las muestras de las PE, los valores de absorción mínima (4\%) son observados a $1150{ }^{\circ} \mathrm{C}$. Este alto valor de absorción de agua refleja en parte el importante contenido en la fracción gruesa. En cuanto al color desarrollado durante la cocción, cabe destacar el blanco desarrollado en las muestras de las PS, el cual puede ser explicado por el alto contenido en caolinita y por la baja concentración en Fe. También ha sido observado un oscurecimiento de las muestras al aumentar la tempera- 
tura, reflejado por la disminución progresiva en los valores de L*. Este oscurecimiento está relacionado con la formación de hematites durante la cocción, a partir de goethita y geles de hierro. Respecto a los minerales de cocción, la presencia de cristobalita en las piezas cocidas de las PP es un aspecto negativo para el uso de estas materias primas, ya que su presencia reduce notablemente la resistencia al choque térmico. Por último, la evolución de la microtextura está caracterizada por el desarrollo de vidrio formando cuellos y filamentos de vidrio. Este material vítreo es el constituyente mayoritario de las piezas cerámicas a partir de la temperatura de vitrificación. A $1200{ }^{\circ} \mathrm{C}$ hay un incremento de la porosidad, probablemente causada por la expansión de los gases inicialmente ocluidos en los poros o procedentes de las reacciones de descomposición de los filosilicatos, causando la deformación macroscópica de los materiales cerámicos.

\section{CONCLUSIONES}

Este estudio ha explorado la posibilidad de usar las pizarras paleozoicas del norte de la provincia de Jaén como una materia prima en la industria cerámica. Desde el punto de vista composicional, estas arcillas están en el rango de las materias primas comúnmente usadas en cerámica estructural. El alto contenido en Fe, debido a la presencia de illita fengítica y/ o hematites, en la mayoría de los materiales estudiados parece ser adecuado para la fabricación de gres rojo. Sólo las muestras de PS tienen una composición mineralógica apropiada para la fabricación de gres blanco, debido a su bajo contenido en Fe.

Las propiedades tecnológicas de las pizarras paleozoicas del norte de la provincia de Jaén indican que son adecuadas para el moldeo por presión. Una vez más, la muestra PS es una excepción, dado que su plasticidad es apropiada para el moldeado por extrusión.

Los valores de contracción lineal y de absorción de agua de las piezas cocidas alrededor de $1000{ }^{\circ} \mathrm{C}$ sugieren que estos materiales son apropiados para la fabricación de algunos tipos de baldosas cerámicas, tales como mayólica o cottoforte. Además, este estudio ha revelado que las pizarras paleozoicas estudiadas cocidas en el intervalo de temperatura comprendido entre $1150{ }^{\circ} \mathrm{C}$ y $1250{ }^{\circ} \mathrm{C}$ pueden ser usadas para manufacturar gres.

Las muestras PE, PB, PC y PCH son apropiadas para la fabricación de gres rojo a temperaturas de $1150{ }^{\circ} \mathrm{C}$ y $1200^{\circ}$ C, debido a la alta vitrificación desarrollada en este rango de temperatura, reflejada en el bajo valor de absorción de agua de menos de $0.2 \%$.

Los materiales de PS son aptos para la fabricación de gres blanco a $1250{ }^{\circ} \mathrm{C}$ con valores de absorción de agua de $0.07 \%$. El alto contenido en caolinita causa el color blanco y el carácter refractario desarrollado durante la cocción. La adición de feldespatos o minerales de litio podrían reducir la tempera- tura óptima de cocción. Los materiales de PP parecen ser más refractarios que el resto de materiales estudiados, ya que no están completamente vitrificados a $1250^{\circ} \mathrm{C}$, presentando un $4 \%$ de absorción de agua.

\section{AGRADECIMIENTOS -}

Los autores agradecen los comentarios constructivos de E. Galán y J. Decleer en una versión anterior de este trabajo. MVV y JJM han obtenido la financiación para llevar a cabo este trabajo a través de la Junta de Andalucía (Grupos de investigación RNM 325 y 179 ) y la Excelentísima Diputación Provincial de Jaén mediante el proyecto de Investigación "Posibilidades de uso cerámico de los minerales y rocas industriales de la provincia de Jaén".

\section{BIBLIOGRAFÍA}

1. B. Fabbri, C. Fiori. «Clays and complementary raw materials for stoneware tiles. Mineral». Petrol. Acta 29-A 535-545 (1985).

2. C. Fiori, B. Fabbri, F. Donati, I Venturi. «Mineralogical composition of the clay bodies used in the Italian tile industry». Appl. Clay Sci. 4 461-473 (1989).

3. M. Dondi. «Clay materials for ceramic tiles from the Sassuolo District (Northern Apennines, Italy). Geology, composition and technological properties». Appl. Clay Sci. 15 337-366.

4. B. Fabbri, M. Dondi.« Mineralogical classification of Italian clay raw materials for production of different ceramics», pp. 45-50 en Proc. 5th Neubrandenburger Industriemineralsymp. 1995

5. D. Martínez Poyatos, F. González Lodeiro, A. Azor, J.F. Simancas. «La estructura de la Zona Centro-lbérica en la región de los Pedroches (Macizo Ibérico Meridional)». Rev. Soc. Geol. España 14 147-160 (2001).

6. E. Barahona. «Arcillas de ladrillería de la provincia de Granada». Tesis Doctoral, Universidad de Granada 1974.

7. E. Galán. Caolines españoles: Geología, Mineralogía y Génesis».Tesis Doctoral, Public. Soc. Ceram. Vidrio1972.

8. F. Nieto. «Las cloritas de la Cordillera Bética». Tesis Doctoral, Universidad de Granada 1984.

9. L. G. Schultz. «Quantitative interpretation of mineralogical composition from x-ray and chemical data for the Pierre shale». U. S. Geol. Surv. Prof. Pap. 391C 31 (1964).

10. J. Parras, C. Sánchez-Jiménez, M. Rodas, F. J. Luque. «Ceramic applications of Middle Ordovician shales from central Spain». Appl. Clay Sci. 11 25-45 (1996).

11. M. J. Orts, A. Escardino, J. L. Amorós, F. Negre. «Microstructural changes during of stoneware floor tiles». Appl. Clay Sci. 8 193-205 (1993).

12. M. Dondi, B. Fabbri, R. Laviano. «Characteristics of the clays utilized in the bricks industry in Apulia and Basilicata (southren Italy)». Miner. Petrogr. Acta 35A 179-189 (1992).

13. H. G. F. Winkler. «Bedeutung der Korngrössenverteilung und des Mineralbestandes von Tonen für die herstellung grobkeramischer Erzeugnisse». Ber. Dtsch. Keram. Ges 31 (10) 337-343 (1954).

14. M. Dondi, B. Fabbri, G. Guarini. «Grain-size distribution of Italian raw materials for building clay products: a reappraisal of the Winkler diagram». Clay Minerals 33 435-442 (1998).

15. J. A. Bain, D. E. Highley. «Regional appraisal of clay resources», pp. 437-447 en A challenge to the clay mineralogist». Proc. Int. Clay Conf. AIPEA, Oxford (USA) 1966

Recibido: 24.01 .03

Aceptado: 21.04 .03 